2016-06-01

\title{
Associations Between Physical and Sedentary Activity Regularity and Sleep in Preschoolers and Kindergartners
}

Kara McRae Duraccio

Follow this and additional works at: https://scholarsarchive.byu.edu/etd

Part of the Psychology Commons

\section{BYU ScholarsArchive Citation}

Duraccio, Kara McRae, "Associations Between Physical and Sedentary Activity Regularity and Sleep in Preschoolers and Kindergartners" (2016). All Theses and Dissertations. 6363.

https://scholarsarchive.byu.edu/etd/6363 
Associations Between Physical and Sedentary Activity Regularity and Sleep in

Preschoolers and Kindergartners

\title{
Kara McRae Duraccio
}

A thesis submitted to the faculty of

Brigham Young University

\begin{abstract}
in partial fulfillment of the requirements for the degree of
\end{abstract}
Master of Science

Chad D. Jensen, Chair

Bruce Bailey

Wendy Birmingham

\author{
Department of Psychology \\ Brigham Young University
}

June 2016

Copyright (C) 2016 Kara McRae Duraccio

All Rights Reserve 


\author{
ABSTRACT \\ Associations Between Physical and Sedentary Activity Regularity and Sleep in \\ Preschoolers and Kindergartners \\ Kara McRae Duraccio \\ Department of Psychology, BYU \\ Master of Science
}

Short sleep duration in childhood has been associated with an increased risk of overweight and obesity. Research suggests that physical activity might mediate this association; however, studies examining associations between physical activity and sleep in young children have reported equivocal findings. A possible explanation for these inconsistencies is that past studies have looked at total physical activity counts rather than examining physical activity regularity. We aim to explore the relationship of regular physical activity, sedentary behavior, and sleep in preschoolers and kindergartners. 131 children (ages 4-6) were included in this study. Each child wore a waist-worn accelerometer for three days and three nights. Waist-worn accelerometers reliably measure sleep and physical activity in children. Associations of regular physical activity, sedentary behavior, and sufficient sleep were determined using logistic regression models. There was no association between the number of days that children engaged in physical activity ( $\geq 60$ minutes) and sufficient sleep. Further, there was no association between the number of days that children engaged in $\geq 20$ minutes of vigorous activity and sufficient sleep. Children who engaged in minimal sedentary activity had greater odds of obtaining sufficient sleep as compared to children who engaged in more sedentary activity.

Keywords: sleep, physical activity, sedentary activity, children 


\section{TABLE OF CONTENTS}

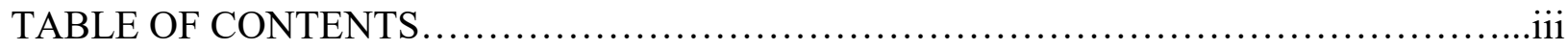

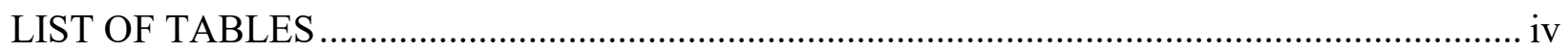

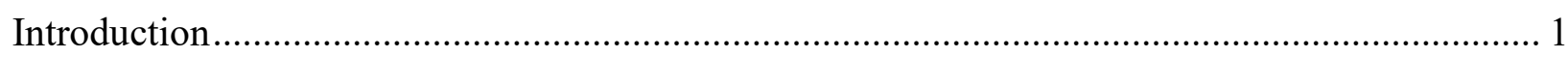

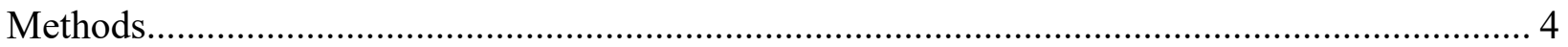

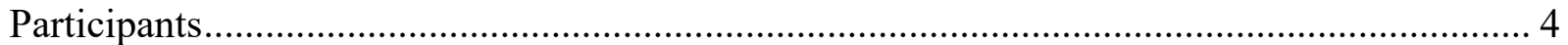

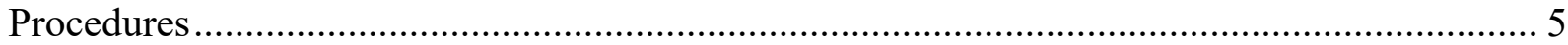

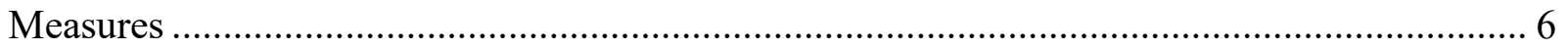

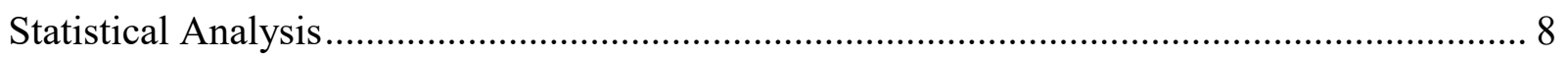

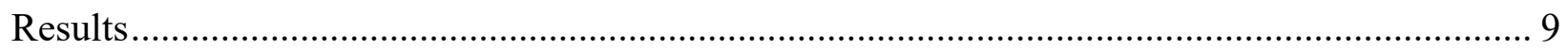

Associations between Physical Activity and Sleep Duration ............................................. 10

Associations between Sedentary Behavior and Sleep Duration ......................................... 11

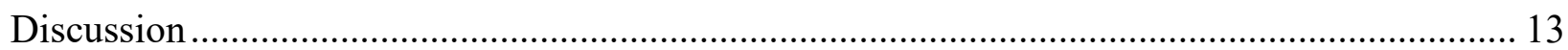

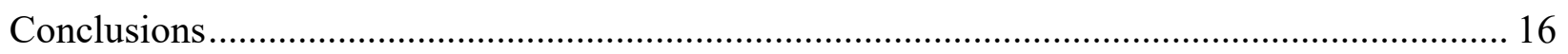

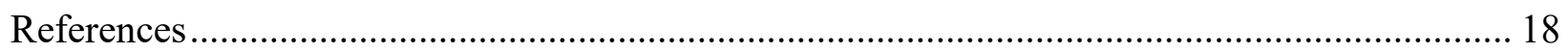




\section{LIST OF TABLES}

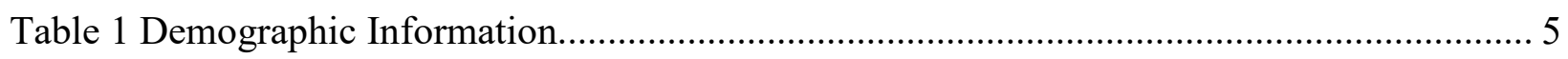

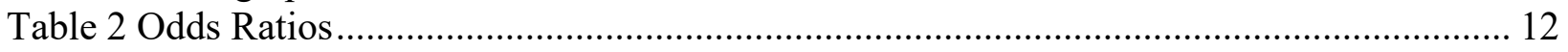


Associations Between Physical and Sedentary Activity Regularity and Sleep in

Preschoolers and Kindergartners

The National Sleep Foundation suggests that children obtain between 9-11 hours of sleep per night, and that preschoolers and kindergartners receive between 10-13 hours of sleep per night (Hirshkowitz et al., 2015). Children who fail to meet these sleep requirements have been shown to experience deficits in decision making, attention, and poorer overall well-being (Nixon et al., 2008). Further, diminished sleep in children has been shown to be associated with a reduction of growth hormones and a dysregulation of appetite hormones (Van Cauter, Spiegel, Tasali, \& Leproult, 2008). Achieving adequate sleep duration is important for the normal functioning of the metabolic and hormonal processes in children.

Short sleep duration has also been shown to be associated with overweight and obesity in children (Ekstedt, Nyberg, Ingre, Ekblom, \& Marcus, 2013; Garaulet et al., 2011; Jarrin, McGrath, \& Drake, 2013; Lumeng et al., 2007; Patel \& Hu, 2008). Several longitudinal studies have been conducted to determine the causal impact of sleep duration on future body mass index (BMI). Two-year-old children who obtained fewer than 11 hours of sleep per night were more likely to be obese at age 7 than children who received more than 12 hours of sleep per night (Agras, Hammer, McNicholas, \& Kraemer, 2004). Additionally, children who were overweight at age nine were reported to sleep an average of 30 fewer minutes per night at ages 3-5 compared to their normal weight peers (Agras et al., 2004). Bell and Zimmerman (2010) examined correlations between sleep duration and weight status five years later in young children (ages 0-4 years) and older children (5-13 years). They found that sleep duration was associated with obesity risk only in younger children. Examining the sleep habits of young children may provide better predictions of future weight status than examining sleep habits in older children. 
The relationship between sleep and childhood obesity may be partially mediated by the physical activity levels of the child. The Centers for Disease Control and Prevention suggest that children engage at least 60 minutes of physical activity daily (2015). Studies show that adolescents who engage in more physical activity have higher odds of obtaining sufficient sleep than those who engage in less physical activity (Foti, Eaton, Lowry, \& McKnight-Ely, 2011; Lang et al., 2013), though some investigators have found this only to be true for higher intensity physical activity (Dworak et al., 2008). However, there is a lack of consensus regarding the effects of physical activity on sleep in younger children. Pesonen and colleagues (2011) found that higher levels of physical activity in young children was associated with decreases in sleep duration and quality. Other sources have found no relationship between sleep duration and physical activity in young children (Kinder, 2010). Additionally, some researchers have found that physical activity duration does not directly mediate the relationship between sleep duration and BMI in young children (Magee, Caputi, \& Iverson, 2014; Williams, Farmer, Taylor, \& Taylor, 2014). As the literature has reported equivocal findings regarding the association between physical activity and sleep duration in younger children, this question merits additional research.

One explanation for such equivocal findings is that total amount of physical activity may not be as critical on impacting sleep duration as regularity of physical activity (i.e., consistent daily activity). Brand and colleagues (2010a) showed that regularity of physical activity, rather than intensity or total time of physical activity, improved sleep patterns in adolescents. In a later study, Brand and colleagues (2010b) found that adolescents who regularly engaged in vigorous exercise had improved sleep quality and duration. Additionally, they reported that adolescent males with less regular physical activity were at an increased risk for reporting sleep complaints. 
Further, it has also been established that less habitual exercise is associated with an increased risk for insomnia in Chinese adolescents (Liu, Uchiyama, Okawa, \& Kurita, 2000).

In 2011, Foti and colleagues aimed to further examine the impact of regular physical activity on sleep duration. Using a large, nationally representative sample $(\mathrm{N}=14,782)$ Foti and colleagues established that adolescents who engaged in at least 60 minutes of physical activity on four or more days during the week had higher odds of obtaining sufficient sleep (i.e. 8 or more hours of sleep) than adolescents who did not engage in 60 minutes of physical activity on any given day. Additionally, prevalence of obtaining sufficient sleep was higher for adolescents who engaged in more than 20 minutes of vigorous activity on 5 or more measurement days. These findings suggest that the effect of physical activity on sleep duration may be additive, such that regular participation in physical activity is more likely to positively impact sleep duration, whereas a single bout of physical activity would be less likely to have salutary effects on sleep duration. To date, no study has examined the relationship between physical activity and sleep in young children.

Further, sedentary behaviors such as television watching and computer usage have been shown to negatively associate with children's sleep duration (Dworak et al., 2008; Van den Bulck, 2004), although the evidence for this relationship is not always clear (M. Y. Chen, Wang, \& Jeng, 2006). As such, Foti and colleagues (2011) also examined the impact of sedentary behaviors on the odds of obtaining sufficient sleep. Interestingly, they found that adolescents who watched four or more hours of television daily were more likely to receive sufficient sleep as compared to adolescents who watched less TV. However, they found that adolescents who used the computer more frequently were less likely to obtain sufficient sleep. Due to these contradictory findings, further research needs to examine the relationship between sedentary 
behaviors and sleep duration, especially within a younger population where less research is available.

While regular physical activity has been shown to be associated with better sleep patterns in adolescents, these findings have not been extended to young children, particularly preschoolers/kindergartners. This is a critical age to study sleep habits, as sleep habits at this age have been shown to predict later BMI (Bell \& Zimmerman, 2010). Further, previous research examining physical activity regularity has relied upon self-report measures for determining physical activity duration and intensity. No previous studies have used objective measures to assess regularity of physical activity. We suspect that this reliance on self-report measures of physical activity paired with only examining total counts of physical activity (rather than examining regularity of physical activity) has contributed to the inconsistency in the literature regarding the relationship between physical activity and sleep. Therefore, we hypothesize that greater regularity of physical activity (measured objectively) among preschoolers and kindergarteners will be associated with increased likelihood of obtaining sufficient sleep. We also hypothesize that greater regularity of vigorous physical activity will be associated with increased likelihood of obtaining sufficient sleep. Finally, we hypothesize that greater regularity of sedentary behavior will be associated with decreased odds of obtaining sufficient sleep.

\section{Methods}

\section{Participants}

Participants $(\mathrm{N}=131)$ included parent/child dyads recruited from a university-based preschool/kindergarten program during the 2012-2013 and 2013-2014 school years. Our sample consisted of children ages four to six ( $M=4.91, S D=0.48 ; 43.5 \%$ female $) .74 \%$ of our sample was Caucasian (see Table 1 for demographic information). All children attending the school were invited to participate in the study through an email sent to their parents by the school study 
staff. Informed consent was gathered from all parents/guardians prior to study enrollment.

Parents received a $\$ 10$ gift card upon the completion of the study.

Table 1 Demographic Information

\begin{tabular}{lcc}
\hline \hline Variables & N & \% \\
\hline Age & & 53.4 \\
4 years old & 70 & 37.4 \\
5 years old & 49 & 2.3 \\
6 years old & 3 & 6.9 \\
Missing & 9 & \\
Sex & & 49.6 \\
Male & 65 & 43.5 \\
Female & 57 & 6.9 \\
Missing & 9 & \\
Race/Ethnicity & & 74.0 \\
Caucasian & 97 & 0.0 \\
African American & 0 & 1.5 \\
Hispanic & 2 & 0.8 \\
Native American & 1 & 1.5 \\
Asian & 2 & 3.8 \\
Other & 5 & 18.3 \\
$\quad$ Missing & 24 & $\boldsymbol{S D}$ \\
\hline & $\boldsymbol{M}$ & 3901.07 \\
\hline Monthly Income & 5800.77 & 4.99 \\
\hline Parent Age & 35.20 & \\
\hline
\end{tabular}

\section{Procedures}

All study procedures were approved by the university preschool/kindergarten and the Brigham Young University institutional review board. Parents provided informed consent and demographic data about their children electronically via an online survey. Children heights were measured with a standard stadiometer (SECA 215) and weights were measured with an electronic scale (SECA 813). Accelerometer-measured sleep and physical activity outcome data was collected across a 3-day period using waist-worn accelerometers (Actigraph GT3X+).

Trained research assistants allowed the participants to handle the accelerometers before receiving verbal assent and placing them on the right hip of all participating children. Accelerometers were placed Monday mornings and were collected Thursday mornings. Children wore the 
accelerometer for 72 consecutive hours and were only asked to remove the accelerometer for swimming and bathing. Instructions for wear were given to the parents to maximize wear time. Data collection occurred in September and October 2012 and 2013 to reduce seasonal confounds.

\section{Measures}

Basic demographic information was gathered from all participants, including date of birth, gender, and ethnicity. After gathering weight and height estimates, Body Mass Index Percentile for age and sex (BMI\%) was calculated for each participant. BMI\% is a moderately reliable indicator of body fat percentage (Mei et al., 2002) and has a moderate inverse relationship with measures of cardiovascular fitness, such that individuals with a higher BMI\% are less likely to be aerobically fit (J. L. Chen, Unnithan, Kennedy, \& Yeh, 2008; Joshi, Bryan, \& Howat, 2012). BMI was first calculated using a standardized formula $(\mathrm{BMI}=[$ weight (kg)]/[height (m)]2; Keys, Fidanza, Karvonen, Kimura, \& Taylor, 1972), and was then converted to an age- and sex-adjusted percentile score using the Center for Disease Control and Prevention BMI percentile calculator (Centers for Disease Control and Prevention, 2010).

Sleep was measured using accelerometers (Actigraph GT3X+) that were worn over the hip for three consecutive days. Sleep data was cleaned and scored using the Actilife 5 software. Sleep time was established by visually marking when the participant's physical activity nearly ceased in the evening and wake time was established by visually marking when a participant engaged in a noticeable amount of physical activity in the morning ("Actigraph accelerometers," 2014). If no movement was recorded during the night, it was assumed that the accelerometer was removed prior to bedtime and the data was excluded. A sleep report was generated for each participant using the sleep algorithm developed by Sadeh and colleagues (1994); the report included key variables such as sleep latency, sleep duration, sleep efficiency, minutes awake 
after sleep onset, the number of awakenings, and the average awakening length. For the purposes of this study, we only used the sleep duration variable, as the majority of previous studies have used sleep duration as their primary variable of interest. Further, the National Sleep Foundation has established a clear cutoff for healthy sleep duration for younger children (Hirshkowitz et al., 2015), but clear cutoffs for the other sleep variables generated have yet to be established. Finally, waist-worn actigraph accelerometers have been shown to provide accurate measures of total sleep duration (Hjorth et al., 2012; Paavonen, Fjällberg, Steenari, \& Aronen, 2002).

Physical activity data was also measured using waist-worn accelerometer data. The ActiGraph GT3X+ uses a solid-state triaxial accelerometer to measure motion on three separate axes ("Actigraph accelerometers," 2014). Waist-worn accelerometer placement provides the greatest accuracy in detecting daytime movement (Cleland et al., 2013). For the present study, accelerometers were initialized to save the movement data in 15-second intervals (epochs; Pate, Almeida, Pfeiffer, \& Dowda, 2006). After children wore the accelerometer for three consecutive days, accelerometer data was downloaded onto ActiLife5 in .agd format. To analyze physical activity, files were converted to a .csv format through ActiLife5. MeterPlus4.3 was used to both clean and score the data. Participants needed to have at least 8 valid hours of recorded physical activity to be considered a valid wear day (60 consecutive minutes of non-movement was defined as an invalid hour; "Actigraph accelerometers," 2014). Physical activity was downloaded in an hourly format, and divided into school-wearing time and home-wearing time. Cut points for children ages 4-6 for sedentary, moderate, and vigorous physical activity were set at 0-419 counts/ $15 \mathrm{~s}$ epoch, $420-841$ counts $/ 15 \mathrm{~s}$ epoch, and $842+$ counts $/ 15 \mathrm{~s}$ epoch, respectively (Pate et al., 2006). 


\section{Statistical Analysis}

All preliminary analyses were performed using STATA 13.0 (StataCorp, 2013).

Preliminary analyses indicated that there were 16 mild outliers across sleep, physical activity, and sedentary behavior observations at each time point. These outliers were fenced to fall within two interquartile ranges above the median. All analyses were run before and after fencing outliers, and no significant differences were observed in the analyses; as such, we retained our fenced outliers to maintain the normality of our data. Preliminary analyses also indicated adequate normality of the primary variables at each time point after fencing the outliers. The data was also found to be linear and homoscedastic.

Regarding nighttime accelerometer data, we found that $13.7 \%$ of our participants were missing data on night one, $7.6 \%$ were missing data on night 2 , and $9.2 \%$ of our participants were missing data on night three. Regarding physical activity data, none of our participants were missing any data on days 1 and 2 , but $13.1 \%$ of our participants were missing data on day 3 . Multiple comparisons between adolescents with complete data and those with missing data did not reveal any significant differences on outcomes of interest. Data were therefore deemed to be missing at random, thus enabling the use of full information maximum likelihood estimation (FIML) within STATA. FIML has been shown to generate unbiased parameter estimates by using all the available data to compute the parameter estimates that would be most likely given the data. FIML has been shown to outperform classical methods of accounting for missing data (Enders \& Bandalos, 2001; Peters \& Enders, 2002).

Independent $t$ tests were used to identify differences in gender on the prevalence of sufficient sleep. Further, age and BMI\% were correlated with sleep outcomes to determine if age or $\mathrm{BMI} \%$ were related to the prevalence of obtaining sufficient sleep. Differences were 
considered significant at $p<0.05$. Sleep duration from the accelerometer report output was used to create a "sufficient sleep" dummy variable; participants who slept at least 10 hours each night were coded as 1 , and participants who slept less than 10 hours each night were coded as 0 (Hirshkowitz et al., 2015). To calculate the likelihood of obtaining sufficient sleep using physical and sedentary activity predictors, three separate multivariate logistic regression models were constructed; all assumptions for conducting a logistic regression were met. Gender was used as a control variable in each model.

The first logistic regression model included total days of achieving at least 60 minutes of physical activity as a predictor (Centers for Disease Control and Prevention, 2015; Foti et al., 2011). Daily physical activity was dummy coded so that 0 signified $<60$ minutes of physical activity engagement, and 1 signified $\geq 60$ minutes of physical activity engagement. The second logistic regression model included total days of achieving $\geq 20$ minutes of vigorous activity as a predictor (Foti et al., 2011); vigorous activity was dummy coded similarly to physical activity. The final logistic regression model examined included sedentary behavior (engaged in at home) as a predictor of obtaining sufficient sleep. As there is no clear cutoff established in the literature for optimal sedentary activity, we dichotomized the sedentary activity data by separating those above and below the bottom quartile of sedentary activity. We selected the bottom quartile as our cutoff as opposed to the median because we hypothesized that individuals with very low levels of sedentary behavior would be most likely to have improved sleep outcomes. We then dummy coding these variables, with 1 suggesting the participant fell within the bottom quartile and 0 suggesting that they fell above the bottom quartile. Finally, to determine whether these associations varied by gender, interaction terms were added to each model. 


\section{Results}

Approximately $41 \%$ of our sample obtained sufficient sleep (10 hours or more per night; Hirshkowitz et al., 2015) across the three-day sampling period. Additionally, $46.6 \%$ of our sample obtained sufficient physical activity ( $\geq 60$ minutes of activity each day; Centers for Disease Control and Prevention, 2015) and 16.03\% of our sample had reduced sedentary behavior (sedentary levels in the bottom quartile) across the three-day sampling period. Average daily sedentary time totaled 7.87 hours. There were no gender differences in those who obtained sufficient sleep, obtained sufficient physical activity, or achieved reduced sedentary activity. Females were more likely to have a longer sleep duration on the second night of sleep as compared to males, $t(120)=-2.10, p<0.05$. Further, boys were more likely to engage in greater minutes of overall physical activity on day $3(t(120)=2.90, p<.01)$ and greater minutes of vigorous activity on day $3(t(12)=2.78, p<.01)$ as compared to girls. There were no sex differences in sleep duration on nights one or three, or physical activity and vigorous activity levels for days one and two. Further, there were no differences in sleep duration, physical activity levels, or vigorous activity levels by age or $\mathrm{BMI} \%$.

\section{Associations between Physical Activity and Sleep Duration}

After running the first logistic regression model, we found that gender did not significantly influence the odds of children obtaining sufficient sleep $(p<0.05)$. Odds of obtaining sufficient sleep were not influenced by engaging in $\geq 60$ minutes of physical activity on any of the assessment days (Table 2). The likelihood ratio for this model was $\chi^{2}(\mathrm{df}=6)=0.84, p$ $=0.84$, suggesting that this model did not fit the data significantly better than a model with no predictors (Satorra \& Bentler, 2001). Upon running our second model, we again found that gender did not significantly influence the odds of children obtaining sufficient sleep. Further, the odds of obtaining sufficient sleep were not influenced by engaging in $\geq 20$ minutes of vigorous 
physical activity on any given number of days. The likelihood ratio for this second model was $\chi^{2}(\mathrm{df}=6)=2.61, p=0.62$, suggesting that this second model did not fit significantly better than a model with no predictors.

\section{Associations between Sedentary Behavior and Sleep Duration}

When attempting to analyze our final model, we were unable to calculate the logistic regression due to limited observations in our reference categories. Therefore, we altered our coding to lower the sleep cutoff from 10 hours to 9.5 hours; given the cutoffs established by the National Sleep Foundation, 9.5 hours "may be appropriate" for preschoolers and kindergartners (Hirshkowitz et al., 2015). Using the methods outlined previously, participants who slept at least 9.5 hours each night were coded as 1 , and participants who slept less than 9.5 hours each night were coded as 0 . Lowering the cutoff from 10 hours to 9.5 hours allowed for adequate number of observations in each reference category. With this new cutoff, we found that the likelihood of obtaining sufficient sleep (9.5 hours) across the three-day measurement period increased significantly with lower sedentary activity across assessment days (i.e., more days with less sedentary behavior increased the likelihood of meeting the sleep criterion; Table 2). The likelihood ratio for this final model was $\chi_{(\mathrm{df}=6)}^{2}=12.12, p<0.007$, suggesting that this final model fit the data significantly better than a model with no predictors. Within this model, gender did not significantly predicted odds of obtaining significant sleep $(p=0.38)$. Further, we determined that the overall effect of sedentary behavior on sufficient sleep was significant $\chi^{2}$ (df $=$ $\left.{ }_{3}\right)=10.81, p<0.01$, such that as the number of days in which a child engaged in minimal sedentary behaviors increased, the odds of obtaining sufficient sleep increased. We then reran our model to include the interaction of gender with days of minimal sedentary behaviors; we 
found that there was no interaction between gender and days of minimal sedentary activity

predicting odds of obtaining sufficient sleep ( $p$ s $>0.05)$.

Table 2 Odds Ratios

Odds of Obtaining Sufficient Sleep ${ }^{a}$ by Physical Activity, Vigorous Activity, and Sedentary Behavior Activity

\section{Odds Ratios (95\% CI)}

\begin{tabular}{ll}
\hline $\begin{array}{l}\text { Days in which physical activity } \geq \mathbf{6 0} \\
\text { minutes }\end{array}$ \\
\hline 0 & Ref \\
1 & $0.81(0.15,4.31)$ \\
2 & $0.88(0.29,2.63)$ \\
3 & Omitted \\
\hline Days in which vigorous activity $\geq \mathbf{2 0}$ & \\
minutes & Ref \\
\hline 0 & $0.42(0.10,1.86)$ \\
1 & $0.69(0.15,3.13)$ \\
2 & $1.06(0.29,3.84)$ \\
3 & \\
\hline Days in which sedentary activity fell & \\
below sample median & Ref \\
\hline 0 & $3.53(1.70,9.25)$ \\
1 & $3.96(1.08,11.49)$ \\
2 & \\
\hline${ }^{a}$ Sufficient sleep was defined as as $\geq 10$ hours of sleep per night $($ Hirshkowitz, 2015) \\
$* p<.01$ & \\
$* * p<.001$ & \\
\hline
\end{tabular}

Finally, we determined the probability of a child obtaining sufficient sleep with 0,1 , or 2 days of of high sedentary behavior. Because no child had three days where sedentary activity fell within the bottom quartile, we were unable to determine the probability of obtaining sufficient sleep with having no days of high sedentary activity. However, participants who had only one day of high sedentary behavior had a 0.56 probability of obtaining sufficient sleep across the three-day measurement period $(p<0.001 ; 95 \%$ CI $[0.26,0.75])$. For each added day of high sedentary behavior, the probability of obtaining sufficient sleep decreased ( 1 day $=0.53, p<$ $0.001,95 \%$ CI $[0.40,0.67] ; 2$ days $=0.22, p<0.001,95 \%$ CI $[0.11,0.33])$. 


\section{Discussion}

We measured sleep duration and physical activity levels among preschoolers and kindergartens over a three-day period, hypothesizing that those with greater regularity of physical activity and vigorous activity and consistent low levels of sedentary behavior would demonstrate greater likelihood of achieving sufficient sleep. Contrary to previous findings (Foti et al., 2011), we found that regular physical activity was not associated with the likelihood of obtaining sufficient sleep. Further, regular participation in vigorous activity was not found to be associated with the likelihood of obtaining sufficient sleep. Our findings resembled recent research findings that demonstrated that physical activity levels were not associated with total sleep duration (Kinder, 2010).

While our finding that regular physical activity does not impact the likelihood of obtaining sufficient sleep is contrary to Foti and colleagues findings in adolescents (2011), it is worth noting that the structure of physical activity differs widely in younger children as opposed to older adolescents. For preschoolers and kindergartners, physical activity is accumulated throughout the day through short bouts of activity (Oliver, Schofield, \& Kolt, 2007), whereas physical activity for adolescents tends to be more deliberate and consolidated. Further, younger children often engage in greater levels of activity throughout the day as compared to adolescents (Sallis, Prochaska, \& Taylor, 2000; Trost et al., 2002). Data suggests that the greatest decline in physical activity occurs between the ages of 13-18 (Sallis, 2000). Further, adolescents tend to participate in greater levels of sedentary activities as compared to younger children (Matthews et al., 2008; Bar-Or, 1999). Because adolescents participate in more sedentary behaviors and engage in less physical activity, deliberate regular physical activity may have a greater impact on the sleep habits of adolescents as opposed to younger children, where physical activity is less deliberate and more natural. 
Interestingly, we found that decreases in sedentary behavior were associated with increased odds of obtaining sufficient sleep. The more days a person spent engaging in less sedentary behaviors, the greater their likelihood of obtaining sufficient sleep across the three-day measurement period. While we did not examine specific sedentary behaviors as Foti and colleagues (i.e., separate analyses for television vs. computer use; 2011), we were able to observe that consistent high levels of sedentary behavior decreased the likelihood of obtaining sufficient sleep. Similar studies have shown that higher levels of sedentary behavior may increase the likelihood of having disturbed sleep (Owens et al., 1999).

Within the last decade children and adolescents have engaged in greater levels of sedentary behaviors, likely due to increased availability to television, video games, handheld devices, and Internet access (Matthews et al., 2008). Recent findings suggest that children and adolescents spend 6-8 hours a day participating in sedentary behaviors (Lou, 2014). Within our sample, preschoolers and kindergartners were observed to engage in nearly eight hours of sedentary activity each day, outside of school. Increased sedentary behaviors have been linked to increased weight gain (Rey-Lopez, Vicente-Rodríguez, Biosca, \& Moreno, 2008) and increased risk for cardiovascular disease (Ford, Kohl, Mokdad, \& Ajani, 2005). Further, findings from this study suggest that increased participation in sedentary behaviors decrease young children's likelihood of obtaining sufficient sleep. Decreases in sleep duration have also been linked to increased risk of obesity (Bell \& Zimmerman, 2010). Our current study found that only $41 \%$ of our sample was achieving sufficient sleep across a three-day period. Therefore, sedentary behaviors, especially in younger children, should be targeted in sleep promotion and obesity prevention efforts. 
Finally, several gender differences were observed in our findings. Specifically, boys within our sample engaged in greater physical activity on the third day of the measurement occasion as compared to girls. This is not unusual, and boys have consistently been found to engage in higher activity levels than girls (Trost et al., 2002). However, there were no gender differences in those who obtained sufficient sleep, obtained sufficient physical activity, or achieved reduced sedentary activity. Further, there was no interaction between gender and days of minimal sedentary activity in influencing the odds of obtaining sufficient sleep. These findings suggest that the benefits of reducing sedentary behaviors are equal amongst males and females.

Findings of this study should be interpreted within the context of several limitations. One limitation was that we measured sleep and physical activity over a relatively brief measurement window ( 3 days), which may not be the most accurate representation of a child's habitual physical and sedentary activity habits. Future studies should measure physical activity and sedentary activity trends over longer period of time, perhaps for a week or more, to allow for a more accurate depiction of physical activity and sedentary activity regularity. Further, nearly all variables of interest (i.e., physical activity, sedentary behavior, and sleep length) were measured through a single device (accelerometers). While it has been established that a waist-worn accelerometer can be used for the dual measurement of sleep length and physical activity counts (Hjorth et al., 2012; Paavonen et al., 2002), using a single method to measure these behaviors limits our construct validity. Future studies should include a variety of measurements to assess for sleep, physical activity, and sedentary behaviors such as actigraphy, sleep diaries, and parent self-report measures. 
Similarly, while actigraphy allowed for the examination of broad sedentary behavior, we were unable to examine the contribution of specific types of sedentary activity (e.g., television viewing, computer usage, video-game usage, etc.) on odds of obtaining sufficient sleep. Further, we were unable to differentiate napping behaviors from sedentary behaviors. Similar to the method employed by Foti and colleageus, (2011), future research should examine specific sedentary behaviors in children and their contribution to overall sleep duration. Finally, our sample was a predominately middle-class Caucasian sample, limiting the generalizability of our findings. Future studies should examine physical activity and sleep habits of various races and cultures.

Several strengths of our study should also be noted. First, our study was one of the first to use objective methods to examine the construct of physical activity regularity. We were able to utilize minimally invasive technology (accelerometers) to measure physical activity, sedentary activity, and sleep duration. Further, despite the limitation of having mono-method bias, using the same device to measure physical activity, sedentary activity, and sleep duration allows for greater comparability between the three constructs of interest, as opposed to utilizing a mix of objective and self-report data. Finally, our study was the first to examine the associations between regular physical activity, sedentary behavior, and sleep duration in a large preschool and kindergartner sample. Sleep habits within this age range are critical to evaluate as they have been shown to influence the development of obesity (Bell \& Zimmerman, 2010).

\section{Conclusions}

Our findings indicate that regular physical activity and regular vigorous activity are not related to the likelihood of preschoolers and kindergartners obtaining sufficient sleep. However, we found that young children who engaged in decreased levels of sedentary behavior were more likely to obtain sufficient sleep as compared to those who were engaged in increased levels of 
sedentary behavior. As decreased sleep within young children has been shown to influence the likelihood of developing obesity (Bell \& Zimmerman, 2010), these findings may suggest that prevention and intervention efforts aimed at reducing the incidence of childhood obesity and other negative outcomes associated with insufficient sleep should include recommendations that target the reduction of sedentary behaviors in young children. 


\section{References}

Actigraph accelerometers. (2014). ActiGraph, LLC. Retrieved from http://www.actigraphcorp.com/

Agras, W. S., Hammer, L. D., McNicholas, F., \& Kraemer, H. C. (2004). Risk factors for childhood overweight: A prospective study from birth to 9.5 years. The Journal of Pediatrics, 145(1), 20-25. doi:http://dx.doi.org/10.1016/j.jpeds.2004.03.023

Bell, J. F., \& Zimmerman, F. J. (2010). Shortened nighttime sleep duration in early life and subsequent childhood obesity. Archives of Pediatrics and Adolescent Medicine, 164(9), $840-845$

Brand, S., Gerber, M., Beck, J., Hatzinger, M., Pühse, U., \& Holsboer-Trachsler, E. (2010a). Exercising, sleep-EEG patterns, and psychological functioning are related among adolescents. World Journal of Biological Psychiatry, 11(2), 129-140. doi:10.3109/15622970903522501

Brand, S., Gerber, M., Beck, J., Hatzinger, M., Pühse, U., \& Holsboer-Trachsler, E. (2010b). High exercise levels are related to favorable sleep patterns and psychological functioning in adolescents: A comparison of athletes and controls. Journal of Adolescent Health, 46(2), 133-141. doi:http://dx.doi.org/10.1016/j.jadohealth.2009.06.018

Centers for Disease Control and Prevention. (2010). Children's BMI tool for schools. Retrieved from http://www.cdc.gov/healthyweight/assessing/bmi/childrens_BMI/tool_for_schools.html

Centers for Disease Control and Prevention. (2015). How much physical activity do children need? Retrieved from http://www.cdc.gov/physicalactivity/basics/children/ 
Chen, J. L., Unnithan, V., Kennedy, C., \& Yeh, C. H. (2008). Correlates of physical fitness and activity in Taiwanese children. International Nursing Review, 55(1), 81-88. doi:10.1111/j.1466-7657.2007.00588.x

Chen, M. Y., Wang, E. K., \& Jeng, Y. J. (2006). Adequate sleep among adolescents is positively associated with health status and health-related behaviors. BMC Public Health, 6(1), 1.

Cleland, I., Kikhia, B., Nugent, C., Boytsov, A., Hallberg, J., Synnes, K., . . Finlay, D. (2013). Optimal placement of accelerometers for the detection of everyday activities. Sensors, 13(7), 9183-9200.

Dworak, M., Wiater, A., Alfer, D., Stephan, E., Hollmann, W., \& Strüder, H. K. (2008). Increased slow wave sleep and reduced stage 2 sleep in children depending on exercise intensity. Sleep Medicine, 9(3), 266-272. doi:10.1016/j.sleep.2007.04.017

Ekstedt, M., Nyberg, G., Ingre, M., Ekblom, Ö., \& Marcus, C. (2013). Sleep, physical activity and BMI in six to ten-year-old children measured by accelerometry: A cross-sectional study. International Journal of Behavioral Nutrition \& Physical Activity, 10(1), 82-91. doi:10.1186/1479-5868-10-82

Enders, C. K., \& Bandalos, D. L. (2001). The relative performance of full information maximum likelihood estimation for missing data in structural equation models. Structural Equation Modeling, 8(3), 430-457.

Ford, E. S., Kohl, H. W., Mokdad, A. H., \& Ajani, U. A. (2005). Sedentary behavior, physical activity, and the metabolic syndrome among US adults. Obesity Research, 13(3), 608614. 
Foti, K. E., Eaton, D. K., Lowry, R., \& McKnight-Ely, L. R. (2011). Sufficient sleep, physical activity, and sedentary behaviors. American Journal of Preventive Medicine, 41(6), 596602. doi:http://dx.doi.org/10.1016/j.amepre.2011.08.009

Garaulet, M., Ortega, F. B., Ruiz, J. R., Rey-López, J. P., Béghin, L., Manios, Y., . . Moreno, L. A. (2011). Short sleep duration is associated with increased obesity markers in European adolescents: Effect of physical activity and dietary habits. The HELENA study. International Journal of Obesity, 35(10), 1308-1317. doi:10.1038/ijo.2011.149

Hirshkowitz, M., Whiton, K., Albert, S. M., Alessi, C., Bruni, O., DonCarlos, L., .. Ware, J. C. (2015). National Sleep Foundation's updated sleep duration recommendations: Final report. Sleep Health, 1(4), 233-243. doi:http://dx.doi.org/10.1016/j.sleh.2015.10.004

Hjorth, M. F., Chaput, J. P., Damsgaard, C. T., Dalskov, S. M., Michaelsen, K. F., Tetens, I., \& SjÖDin, A. (2012). Measure of sleep and physical activity by a single accelerometer: Can a waist-worn Actigraph adequately measure sleep in children? Sleep \& Biological Rhythms, 10(4), 328-335. doi:10.1111/j.1479-8425.2012.00578.x

Jarrin, D. C., McGrath, J. J., \& Drake, C. L. (2013). Beyond sleep duration: Distinct sleep dimensions are associated with obesity in children and adolescents. International Journal of Obesity, 37(4), 552-558. doi:10.1038/ijo.2013.4

Joshi, P., Bryan, C., \& Howat, H. (2012). Relationship of body mass index and fitness levels among schoolchildren. Journal of Strength and Conditioning Research, 26(4), 10061014. doi:10.1519/JSC.0b013e31822dd3ac

Keys, A., Fidanza, F., Karvonen, M. J., Kimura, N., \& Taylor, H. L. (1972). Indices of relative weight and obesity. Journal of Chronic Diseases, 25(6-7), 329-343. doi:http://dx.doi.org/10.1016/0021-9681(72)90027-6 
Kinder, J. R. (2010). Investigating the relationship between physical activity and sleep in children, using accelerometers. (Doctoral dissertation, Univ. of Calif., San Francisco and San Francisco State Univ.).

Lang, C., Brand, S., Feldmeth, A. K., Holsboer-Trachsler, E., Pühse, U., \& Gerber, M. (2013). Increased self-reported and objectively assessed physical activity predict sleep quality among adolescents. Physiology \& Behavior, 120(0), 46-53. doi:http://dx.doi.org/10.1016/j.physbeh.2013.07.001

Liu, X., Uchiyama, M., Okawa, M., \& Kurita, H. (2000). Prevalence and correlates of selfreported sleep problems among Chinese adolescents. Sleep, 23(1), 27-34.

Lou, D. W. (2014). Sedentary behaviors and youth: Current trends and the impact on health. Retrieved from http://activelivingresearch.org/sedentaryreview

Lumeng, J. C., Somashekar, D., Appugliese, D., Kaciroti, N., Corwyn, R. F., \& Bradley, R. H. (2007). Shorter sleep duration is associated with increased risk for being overweight at ages 9 to 12 years. Pediatrics, 120(5), 1020-1029. doi:10.1542/peds.2006-3295

Magee, C., Caputi, P., \& Iverson, D. (2014). Lack of sleep could increase obesity in children and too much television could be partly to blame. Acta Paediatrica, 103(1), e27-e31. doi:10.1111/apa.12447

Matthews, C. E., Chen, K. Y., Freedson, P. S., Buchowski, M. S., Beech, B. M., Pate, R. R., \& Troiano, R. P. (2008). Amount of time spent in sedentary behaviors in the United States, 2003-2004. American Journal of Epidemiology, 167(7), 875-881.

Mei, Z., Grummer-Strawn, L. M., Pietrobelli, A., Goulding, A., Goran, M. I., \& Dietz, W. H. (2002). Validity of body mass index compared with other body-composition screening 
indexes for the assessment of body fatness in children and adolescents. American Journal of Clinical Nutrition, 75(6), 978-985.

Nixon, G. M., Thompson, J. M., Han, D. Y., Becroft, D. M., Clark, P. M., Robinson, E., .. . Mitchell, E. A. (2008). Short sleep duration in middle childhood: Risk factors and consequences. Sleep, 31(1), 71.

Oliver, M., Schofield, G. M., \& Kolt, G. S. (2007). Physical activity in preschoolers. Sports Medicine, 37(12), 1045-1070.

Paavonen, E. J., Fjällberg, M., Steenari, M. R., \& Aronen, E. T. (2002). Actigraph placement and sleep estimation in children. Sleep, 25(2), 235-237.

Pate, R. R., Almeida, K. L., Pfeiffer, K. A., \& Dowda, M. (2006). Validation and calibration of an accelerometer in preschool children. Obesity, 14(11), 2000-2006. doi:10.1038/oby.2006.234

Patel, S. R., \& Hu, F. B. (2008). Short sleep duration and weight gain: A systematic review. Obesity, 16(3), 643-653. doi:10.1038/oby.2007.118

Pesonen, A., Sjöstén, N. M., Matthews, K. A., Heinonen, K., Martikainen, S., Kajantie, E., . . Räikkönen, K. (2011). Temporal associations between daytime physical activity and sleep in children. PLoS ONE, 6(8), 1-6. doi:10.1371/journal.pone.0022958

Peters, C. L. O., \& Enders, C. (2002). A primer for the estimation of structural equation models in the presence of missing data: Maximum likelihood algorithms. Journal of Targeting, Measurement and Analysis for Marketing, 11(1), 81-95.

Rey-Lopez, J. P., Vicente-Rodríguez, G., Biosca, M., \& Moreno, L. A. (2008). Sedentary behaviour and obesity development in children and adolescents. Nutrition, Metabolism and Cardiovascular Diseases, 18(3), 242-251. 
Sadeh, A., Sharkey, K. M., \& Carskadon, M. A. (1994). Activity-based sleep-wake identification: An empirical test of methodological issues. Sleep, 17(3), 201-207.

Sallis, J. F. (2000). Age-related decline in physical activity: A synthesis of human and animal studies. Medicine and Science in Sports and Exercise, 32(9), 1598-1600.

Sallis, J. F., Prochaska, J. J., \& Taylor, W. C. (2000). A review of correlates of physical activity of children and adolescents. Medicine and Science in Sports and Exercise, 32(5), 963975.

StataCorp. (2013). Release 13. Statistical Software. College Station. Texas, USA: StataCorp LP. Trost, S. G., Pate, R. R., Sallis, J. F., Freedson, P. S., Taylor, W. C., Dowda, M., \& Sirard, J. R. (2002). Age and gender differences in objectively measured physical activity in youth. Medicine and Science in Sports and Exercise, 34(2), 350-355.

Van Cauter, E., Spiegel, K., Tasali, E., \& Leproult, R. (2008). Metabolic consequences of sleep and sleep loss. Sleep Medicine, 9, S23-S28.

Van den Bulck, J. (2004). Television viewing, computer game playing, and Internet use and selfreported time to bed and time out of bed in secondary-school children. Sleep, 27(1), 101104.

Williams, S. M., Farmer, V. L., Taylor, B. J., \& Taylor, R. W. (2014). Do more active children sleep more? A repeated cross-sectional analysis using accelerometry. PLoS ONE, 9(4), 18. doi:10.1371/journal.pone.0093117 